\title{
An Initial Boundary Value Problem for the Zakharov Equation
}

\author{
Quankang Yang ${ }^{1,2}$ and Charles $B \mathbf{u}^{3}$ \\ ${ }^{1}$ Institute of Mathematics, School of Mathematical Sciences, Nanjing Normal University, Nanjing 210008, China \\ ${ }^{2}$ Department of Mathematics, Nanjing Xiaozhuang College, Nanjing 210008, China \\ ${ }^{3}$ Department of Mathematics, Wellesley College, Wellesley, MA 02481, USA
}

Correspondence should be addressed to Charles Bu; cbu@wellesley.edu

Received 15 February 2013; Accepted 20 June 2013

Academic Editor: Nikolai A. Kudryashov

Copyright (C) 2013 Q. Yang and C. Bu. This is an open access article distributed under the Creative Commons Attribution License, which permits unrestricted use, distribution, and reproduction in any medium, provided the original work is properly cited.

This paper studies an inhomogeneous initial boundary value problem for the one-dimensional Zakharov equation. Existence and uniqueness of the global strong solution are proved by Galerkin's method and integral estimates.

\section{Introduction}

In this paper, we consider the following inhomogeneous initial boundary value problem for the Zakharov equations in one dimension:

$$
\begin{gathered}
i E_{t}+E_{x x}=n E, \quad x \in(a, b), t>0, \\
n_{t t}-n_{x x}=\left(|E|^{2}\right)_{x x}, \quad x \in(a, b), t>0, \\
E(t, a)=E(t, b)=0, \quad n(t, a)=q_{1}(t), \\
n(t, b)=q_{2}(t), \quad t \geqslant 0, \\
E(0, x)=E_{0}(x), \quad n(0, x)=n_{0}(x), \\
n_{t}(0, x)=n_{1}(x), \quad x \in(a, b) .
\end{gathered}
$$

Zakharov equations play an important role in the turbulence theory for plasma waves and resemble closely to the nonlinear Schrödinger equations. There has been extensive study both theoretically and numerically on these equations (e.g., see [1-17]). Most investigation have been focused on the Cauchy problem of the system of Zakharov equations (SZEs), sometimes with a homogeneous boundary condition. It is well known that Zakharov equations possess 1D soliton solutions. Some numerical experiments suggest that the solutions of 2D and 3D SZE may become singular in finite time $[7,18]$. Global existence of solutions of the $m$-dimensional SZE $(m \geqslant 2)$ has been only proved for small initial data $[5,6]$.
It has been shown that solutions with large initial data may blow up in finite time [8].

The system (1)-(2) above describes the interaction of a Langmuir wave and an ion acoustic wave in a plasma (Dendy [19] and Bellan [20]). Here, $E(t, x)$ is an unknown complex vector-valued function that denotes a slowly varying envelope of a highly oscillatory electric field. Meanwhile, $n(t, x)$ is an unknown real function that denotes the fluctuation in the ion density about its equilibrium value (see $[19,21])$. We assume that $q_{1}(t), q_{2}(t)$ are given smooth functions.

Let $Q(t, x)$ be any $C^{4}$ function on $X=[0, \infty) \times(a, b)$ with compact support satisfying $Q(t, a)=q_{1}(t), Q(t, b)=q_{2}(t)$, and we define

$$
V(t, x)=n(t, x)-Q(t, x), \quad G(t, x)=-Q_{t t}+Q_{x x} .
$$

Thus, the problem (1)-(4) is equivalent to

$$
\begin{gathered}
i E_{t}+E_{x x}=(V+Q) E, \\
V_{t t}-V_{x x}=\left(|E|^{2}\right)_{x x}+G, \\
E(t, a)=E(t, b)=0, \quad V(t, a)=V(t, b)=0, \\
E(0, x)=E_{0}(x), \quad V(0, x)=v_{0}(x), \\
V_{t}(0, x)=v_{1}(x) .
\end{gathered}
$$

In Section 2, we obtain the existence of a local weak solution via Galerkin's method and the principle of compactness. 
In Section 3, we derive estimates of higher-order derivatives of Galerkin's approximate solution to obtain the existence and uniqueness of the local strong solution. In Section 3, we prove the existence and uniqueness of the global strong solution.

\section{Existence of a Local Strong Solution}

We first work on Galerkin's approximation solution for the problem (6)-(9) by choosing the basic functions $\left\{w_{j}(x)\right\}$ as follows:

$$
\begin{gathered}
-w_{j x x}=\lambda_{j} w_{j},\left.\quad w_{j}\right|_{\partial \Omega}=0, \\
w_{j} \in H^{2}(\Omega), \quad\left\|w_{j}\right\|=1, \quad j=1,2, \ldots N .
\end{gathered}
$$

The approximate solution for the problem (6)-(9) can be written as

$$
\begin{aligned}
& E_{N}(t, x)=\sum_{j=1}^{N} p_{j N}(t) w_{j}, \\
& V_{N}(t, x)=\sum_{j=1}^{N} q_{j N}(t) w_{j} .
\end{aligned}
$$

According to Galerkin's method, these undetermined coefficients $p_{j N}(t), q_{j N}(t)$ must satisfy the following initial value problem for a system of ordinary differential equations:

$$
\begin{aligned}
& i\left(E_{N t}, w_{j}\right)+\left(E_{N x x}, w_{j}\right)=\left(E_{N} V_{N}, w_{j}\right)+\left(E_{N} Q, w_{j}\right) \\
& \left.\left(V_{N t t}, w_{j}\right)-\left(V_{N x x}, w_{j}\right)=\left(\left|E_{N}\right|^{2}\right)_{x x}, w_{j}\right)+\left(G, w_{j}\right)
\end{aligned}
$$

with $p_{j N}(0)=\left(E_{0}(x), w_{j}\right), q_{j N}(0)=\left(v_{0}(x), w_{j}\right), q_{j N}^{\prime}(0)=$ $\left(v_{1}(x), w_{j}\right)$ and

$$
E_{N}(0, x)=\sum_{j=1}^{N} p_{j N}(0) w_{j}, \quad V_{N}(0, x)=\sum_{j=1}^{N} q_{j N}(0) w_{j}
$$

To obtain existence of a local weak solution, we need the following lemmas.

Lemma 1. Assume that $E_{0}(x) \in L^{2}$, then $\left\|E_{N}(t, x)\right\|^{2} \leqslant$ $\left\|E_{0}(x)\right\|^{2}$.

Proof. We multiply (13) by $\overline{p_{j N}(t)}$ and sum up in $j$ to obtain

$$
i\left(E_{N t}, E_{N}\right)+\left(E_{N x x}, E_{N}\right)=\left(E_{N} V_{N}, E_{N}\right)+\left(E_{N} Q, E_{N}\right) .
$$

Since $(d / d t)\left\|E_{N}\right\|^{2}=2 \operatorname{Re}\left(E_{N t}, E_{N}\right)$, taing imaginary parts of (16) yields $\operatorname{Re}\left(E_{N t}, E_{N}\right)=0$. Therefore, $\left\|E_{N}\right\|^{2}$ is a constant, and (16) is proved.

From Gagliardo-Nirenberg inequality [22], one has (if $u \in$ $\left.H^{2} \cap H_{0}^{1}\right)$

$$
\|u\|_{L^{\infty}} \leqslant C\|u\|_{H^{1}}^{1 / 2}\|u\|^{1 / 2}, \quad\|u\|_{L^{4}} \leqslant C\left\|u_{x}\right\|^{1 / 4}\|u\|^{3 / 4},
$$

which will be used in the following estimates.
Lemma 2. Let $E_{0}(x) \in H^{2} \cap H_{0}^{1}$, let $v_{0}(x) \in H_{0}^{1}$, let $v_{1}(x) \in L^{2}$, and let $Q(t, x) \in C^{2}\left(0, T ; H^{2}\right)$. Then, there is $T_{0}>0$ such that

$$
\|\Phi\|^{2}=\left\|E_{N x}\right\|^{2}+\left\|V_{N t}\right\|^{2}+\left\|V_{N x}\right\|^{2}+\left\|E_{N t}\right\|^{2} \leqslant C\left(T_{0}\right),
$$

for any $0 \leqslant t \leqslant T_{0}$, where $C$ is a positive constant depending only on data and $T_{0}$.

Proof. We multiply (13) by $\overline{p_{j N}^{\prime}(t)}$, multiply (14) by $q_{j N}^{\prime}(t)$, and sum up in $j$ to get

$$
i\left(E_{N t}, E_{N t}\right)+\left(E_{N x x}, E_{N t}\right)=\left(E_{N} V_{N}, E_{N t}\right)+\left(E_{N} Q, E_{N t}\right),
$$

$$
\left(V_{N t t}, V_{N t}\right)-\left(V_{N x x}, V_{N t}\right)=\left(\left(\left|E_{N}\right|^{2}\right)_{x x}, V_{N t}\right)+\left(G, V_{N t}\right) .
$$

By taking the imaginary parts of (19), we get

$$
-\frac{1}{2} \frac{d}{d t}\left\|E_{N x}\right\|^{2}=\frac{1}{2} \int_{\Omega} V_{N} \frac{d}{d t}\left|E_{N}\right|^{2} d x+\frac{1}{2} \int_{\Omega} Q \frac{d}{d t}\left|E_{N}\right|^{2} d x .
$$

From (20), we have

$$
\begin{aligned}
\frac{1}{2} \frac{d}{d t}\left\|V_{N t}\right\|^{2}+\frac{1}{2} \frac{d}{d t}\left\|V_{N x}\right\|^{2} \\
\quad=\int_{a}^{b}\left(\left|E_{N}\right|^{2}\right)_{x x} V_{N t} d x+\int_{a}^{b} G V_{N t} d x .
\end{aligned}
$$

Combining (21) and (22), we find

$$
\begin{aligned}
& \frac{d}{d t}\left(\left\|E_{N x}\right\|^{2}+\left\|V_{N t}\right\|^{2}+\left\|V_{N x}\right\|^{2}\right) \\
&=-\int_{a}^{b} V_{N} \frac{d}{d t}\left|E_{N}\right|^{2} d x-\int_{a}^{b} \mathrm{Q} \frac{d}{d t}\left|E_{N}\right|^{2} d x \\
&+2 \int_{a}^{b}\left(\left|E_{N}\right|^{2}\right)_{x x} V_{N t} d x+2 \int_{a}^{b} G V_{N t} d x \\
&=-2 \operatorname{Re} \int_{a}^{b} E_{N} \overline{E_{N t}} V_{N} d x-2 \operatorname{Re} \int_{a}^{b} E_{N} \overline{E_{N t}} Q d x \\
&+4 \operatorname{Re} \int_{a}^{b} E_{N} \overline{E_{N x x}} V_{N t} d x+4 \int_{a}^{b}\left|E_{N x}\right|^{2} V_{N t} d x \\
&+2 \int_{a}^{b} G V_{N t} d x .
\end{aligned}
$$

We multiply (13) by $-\lambda_{j} \overline{p_{j N}(t)}$ and sum up $j$ to obtain

$$
\left\|E_{N x x}\right\|^{2}=\left(E_{N} V_{N}, E_{N x x}\right)+\left(E_{N} Q, E_{N x x}\right)-i\left(E_{N t}, E_{N x x}\right) .
$$

Therefore,

$$
\begin{aligned}
\left\|E_{N x x}\right\| & \leqslant\left\|E_{N} V_{N}+E_{N} Q-i E_{N t}\right\| \\
& \leqslant\left\|E_{N} V_{N}\right\|+\left\|E_{N} Q\right\|+\left\|E_{N t}\right\| .
\end{aligned}
$$


Differentiating (13) in $t$, we have

$$
\begin{aligned}
i\left(E_{N t t}, w_{j}\right)+\left(E_{N t x x}, w_{j}\right) & \\
= & \left(E_{N t} V_{N}, w_{j}\right)+\left(E_{N} V_{N t}, w_{j}\right) \\
& +\left(E_{N t} Q, w_{j}\right)+\left(E_{N} Q_{t}, w_{j}\right), \\
i\left(E_{N t t},\right. & \left.E_{N t}\right)+\left(E_{N t x x}, E_{N t}\right) \\
= & \left(E_{N t} V_{N}, E_{N t}\right)+\left(E_{N} V_{N t}, E_{N t}\right) \\
& +\left(E_{N t} Q, E_{N t}\right)+\left(E_{N} Q_{t}, E_{N t}\right) .
\end{aligned}
$$

By taking the imaginary parts of (27), we obtain the following inequalities:

$$
\begin{aligned}
\frac{d}{d t}\left\|E_{N t}\right\|^{2} & =2 \operatorname{Im}\left(E_{N} V_{N t}, E_{N t}\right)+2 \operatorname{Im}\left(E_{N} Q_{t}, E_{N t}\right) \\
& \leqslant\left\|E_{N}\right\|_{L^{\infty}}\left(\left\|V_{N t}\right\|^{2}+2\left\|E_{N t}\right\|^{2}+\left\|Q_{t}\right\|^{2}\right) .
\end{aligned}
$$

From (23), we have

$$
\begin{aligned}
& \frac{d}{d t}\left(\left\|E_{N x}\right\|^{2}+\left\|V_{N t}\right\|^{2}+\left\|V_{N x}\right\|^{2}\right) \\
& \leqslant 2\left|\int_{a}^{b} E_{N} \overline{E_{N t}} V_{N} d x\right|+2\left|\int_{a}^{b} E_{N} \overline{E_{N t}} Q d x\right| \\
& \quad+4\left|\int_{a}^{b} E_{N} \overline{E_{N x x}} V_{N t} d x\right|+\left.4\left|\int_{a}^{b}\right| E_{N x}\right|^{2} V_{N t} d x \mid \\
& \quad+2\left|\int_{a}^{b} G V_{N t} d x\right| \\
& \leqslant 2\left\|E_{N}\right\|_{L^{\infty}} \int_{a}^{b}\left|\overline{E_{N t}} V_{N}\right| d x+2\left\|E_{N}\right\|_{L^{\infty}} \int_{a}^{b}\left|\overline{E_{N t}} Q\right| d x \\
& \quad+4\left\|E_{N}\right\|_{L^{\infty}} \int_{a}^{b}\left|\overline{E_{N x x}} V_{N t}\right| d x+4 \int_{a}^{b}\left|E_{N x}\right|^{2}\left|V_{N t}\right| d x \\
& \quad+2 \int_{a}^{b}\left|G V_{N t}\right| d x .
\end{aligned}
$$

Combining (28), (29), and (25), we get

$$
\begin{gathered}
\frac{d}{d t}\left(\left\|E_{N x}\right\|^{2}+\left\|V_{N t}\right\|^{2}+\left\|V_{N x}\right\|^{2}+\left\|E_{N t}\right\|^{2}\right) \\
\leqslant\left\|E_{N}\right\|_{L^{\infty}}\left(2 \int_{a}^{b}\left|\overline{E_{N t}} V_{N}\right| d x+2 \int_{a}^{b}\left|\overline{E_{N t}} Q\right| d x\right. \\
+4 \int_{a}^{b}\left|\overline{E_{N x x}} V_{N t}\right| d x+\left\|V_{N t}\right\|^{2} \\
\left.+2\left\|E_{N t}\right\|^{2}+2\left\|Q_{t}\right\|^{2}\right) \\
+4 \int_{a}^{b}\left|E_{N x}\right|^{2}\left|V_{N t}\right| d x+\|G\|^{2}+\left\|V_{N t}\right\|^{2} \\
\leqslant\left\|E_{N}\right\|_{L^{\infty}}\left(4\left\|E_{N t}\right\|^{2}+\left\|V_{N}\right\|^{2}+\|Q\|^{2}+2\left\|E_{N x x}\right\|^{2}\right. \\
\left.+3\left\|V_{N t}\right\|^{2}+2\left\|Q_{t}\right\|^{2}\right) \\
+2\left\|E_{N x}\right\|_{L^{4}}^{4}+3\left\|V_{N t}\right\|^{2}+\|G\|^{2} .
\end{gathered}
$$

By Gagliardo-Nirenberg's inequality with $m=1$, we obtain the following estimates:

$$
\begin{gathered}
\left\|E_{N}\right\|_{L^{\infty}} \leqslant C\left\|E_{N}\right\|_{H^{1}}^{1 / 2}\left\|E_{N}\right\|^{1 / 2} \leqslant C\left\|E_{N x}\right\|^{1 / 2}, \\
\left\|E_{N} V_{N}\right\|^{2}=\int_{a}^{b}\left|E_{N} V_{N}\right|^{2} d x \leqslant \frac{1}{2}\left\|E_{N}\right\|_{L^{4}}^{4}+\frac{1}{2}\left\|V_{N}\right\|_{L^{4}}^{4} \\
\leqslant C\left\|E_{N x}\right\|\left\|E_{N}\right\|^{3}+C\left\|V_{N x}\right\|\left\|V_{N}\right\|^{3} \\
\leqslant C\left\|E_{N x}\right\|+C\left\|V_{N x}\right\|^{4}, \\
\left\|E_{N} Q\right\|^{2} \leqslant C\left\|E_{N x}\right\|+C\left\|Q_{x}\right\|^{4} .
\end{gathered}
$$

From (25), (31), (32), and (33), we have

$$
\begin{aligned}
& \left\|E_{N x x}\right\|^{2} \leqslant\left\|E_{N} V_{N}+E_{N} Q-i E_{N t}\right\|^{2} \\
& \leqslant C\left\|E_{N x}\right\|+C\left\|V_{N x}\right\|^{4}+C\left\|Q_{x}\right\|^{4}+3\left\|E_{N t}\right\|^{2}, \\
& \left\|E_{N x}\right\|_{L^{4}}^{4} \\
& \leqslant C\left\|E_{N x x}\right\|\left\|E_{N x}\right\|^{3} \leqslant C \frac{1+\left\|E_{N x x}\right\|^{2}}{2}\left\|E_{N x}\right\|^{3} \\
& \leqslant C\left\|E_{N x}\right\|^{3}+C\left\|E_{N x}\right\|^{4}+C\left\|E_{N x}\right\|^{6} \\
& +C\left\|V_{N x}\right\|^{8}+\frac{3}{2} C\left\|E_{N t x}\right\|^{4}+C\left\|Q_{x}\right\|^{8}
\end{aligned}
$$

with $\left\|V_{N}\right\| \leqslant C\left\|V_{N x}\right\|$. Put the above inequalities into (30), and use Young's inequality $a^{1 / p} b^{1 / q} \leqslant(a / p)+(b / q)$ to obtain

$$
\begin{gathered}
\frac{d}{d t}\|\Phi\|^{2} \frac{d}{d t}\left(\left\|E_{N x}\right\|^{2}+\left\|V_{N t}\right\|^{2}+\left\|V_{N x}\right\|^{2}+\left\|E_{N t}\right\|^{2}\right) \\
\leqslant C\left\|E_{N x}\right\|^{1 / 2}\left[4\left\|E_{N t}\right\|^{2}+\left\|V_{N}\right\|^{2}+\|Q\|^{2}\right. \\
+C\left\|E_{N x}\right\|+C\left\|V_{N x}\right\|^{4}+C\left\|Q_{x}\right\|^{4} \\
\left.+6\left\|E_{N t}\right\|^{2}+3\left\|V_{N t}\right\|^{2}+2\left\|Q_{t}\right\|^{2}\right] \\
+C\left\|E_{N x}\right\|^{3}+C\left\|E_{N x}\right\|^{4}+C\left\|V_{N x}\right\|^{6}+C\left\|V_{N x}\right\|^{8} \\
+3\left\|E_{N t}\right\|^{4}+C\left\|Q_{x}\right\|^{8}+3\left\|V_{N t}\right\|^{2}+\|G\|^{2} \\
\leqslant C\left(\left\|E_{N x}\right\|^{2}+\left\|V_{N t}\right\|^{2}+\left\|V_{N x}\right\|^{2}+\left\|E_{N t}\right\|^{2}\right)^{4} \\
+C \leqslant C\|\Phi\|^{4}+C .
\end{gathered}
$$

Hence, there exist $T_{0}>0$ such that $\|\Phi\|^{2} \leqslant C\left(T_{0}\right)$ for any $0 \leqslant t \leqslant T_{0}$. Here, $C$ only depends on data and $T_{0}$ but not N.

Lemma 3. Under the conditions of Lemma 2, there exist a positive constant $T_{0}$, such that

$$
\begin{gathered}
\left\|E_{N x x}\right\|^{2} \leqslant C\left(T_{0}\right), \quad\left\|V_{N}\right\|^{2} \leqslant C\left(T_{0}\right), \\
\left\|E_{N}\right\|_{L^{\infty}}^{2} \leqslant C\left(T_{0}\right), \quad\left\|E_{N x}\right\|_{L^{4}}^{4} \leqslant C\left(T_{0}\right), \\
\left\|\left(\left|E_{N}\right|^{2}\right)_{x x}\right\|^{2} \leqslant C\left(T_{0}\right),
\end{gathered}
$$


for any $0 \leqslant t \leqslant T_{0}$, and $C$ is a positive constant that depends only on data and $T_{0}$.

Proof. By Lemma 2 and (31), (34), and (35), we have

$$
\begin{gathered}
\left\|E_{N x x}\right\|^{2} \leqslant C\left(T_{0}\right), \\
\left\|E_{N}\right\|_{L^{\infty}}^{2} \leqslant C\left(T_{0}\right), \\
\left\|E_{N x}\right\|_{L^{4}}^{4} \leqslant C\left(T_{0}\right), \\
\left\|\left(\left|E_{N}\right|^{2}\right)_{x x}\right\|=\left\|\left(E_{N} \overline{E_{N}}\right)_{x x}\right\| \\
=\left\|E_{N} \overline{E_{N x x}}+\overline{E_{N}} E_{N x x}+2\left|E_{N}\right|^{2}\right\| \\
\leqslant 2+\left\|E_{N}\right\|_{L \infty}^{2}\left\|E_{N x x}\right\|^{2}+\left\|E_{N x}\right\|_{L^{4}}^{4} \leqslant C\left(T_{0}\right) .
\end{gathered}
$$

Lemma 4. Assume that $E_{0}(x) \in H^{4} \cap H_{0}^{1}, v_{0}(x) \in H^{2} \cap H_{0}^{1}$, $v_{1}(x) \in H^{1}$, and $Q(t, x) \in C^{2}\left(0, T ; H^{4}\right)$, then there exist a positive constant $T_{0}$ such that

$$
\begin{gathered}
\|\Psi\|^{2}=\left\|E_{N t x}\right\|^{2}+\left\|V_{N t t}\right\|^{2}+\left\|V_{N t x}\right\|^{2}+\left\|E_{N t t}\right\|^{2} \leqslant C\left(T_{0}\right), \\
\left\|V_{N x x}\right\|^{2} \leqslant C\left(T_{0}\right)
\end{gathered}
$$

for any $0 \leqslant t \leqslant T_{0}$, where $C$ is a positive constant that depends only on data and $T_{0}$.

Proof. By differentiating (13) twice in $t$, we have

$$
\begin{aligned}
& i\left(E_{N t t t}, w_{j}\right)+\left(E_{N t t x x}, w_{j}\right) \\
& =\left(\left(E_{N} V_{N}\right)_{t t}, w_{j}\right)+\left(\left(E_{N} Q\right)_{t t}, w_{j}\right) .
\end{aligned}
$$

Multiply (40) by $\overline{p_{j N}^{\prime \prime}(t)}$, and sum up in $j$ to obtain

$$
\begin{aligned}
& i\left(E_{N t t t}, E_{N t t}\right)+\left(E_{N t t x x}, E_{N t t}\right) \\
& =\left(\left(E_{N} V_{N}\right)_{t t}, E_{N t t}\right)+\left(\left(E_{N} Q\right)_{t t}, E_{N t t}\right) .
\end{aligned}
$$

Taking the imaginary parts of (41), we have

$$
\begin{aligned}
& \frac{1}{2} \frac{d}{d t}\left\|E_{N t t}\right\|^{2} \\
& \leqslant\left|\left(E_{N t t} V_{N}, E_{N t t}\right)\right|+2\left|\left(E_{N t} V_{N t}, E_{N t t}\right)\right| \\
& +\left|\left(E_{N} V_{N t t}, E_{N t t}\right)\right|+\left|\left(E_{N t t} Q, E_{N t t}\right)\right| \\
& \quad+2\left|\left(E_{N t} Q_{t}, E_{N t t}\right)\right|+\left|\left(E_{N} Q_{t t}, E_{N t t}\right)\right| \\
& \leqslant\left\|V_{N}\right\|_{L^{\infty}}\left\|E_{N t t}\right\|^{2}+2\left\|V_{N t} E_{N t} E_{N t t}\right\|_{L^{1}} \\
& +\left\|E_{N}\right\|_{L^{\infty}}\left\|V_{N t t}\right\|\left\|E_{N t t}\right\| \\
& +\|Q\|_{L^{\infty}}\left\|E_{N t t}\right\|^{2}+2\left\|Q_{t}\right\|_{L^{\infty}}\|\| E_{N t}\|\| E_{N t t} \| \\
& +\left\|Q_{t t}\right\|_{L^{\infty}}\left\|E_{N}\right\|\left\|E_{N t t}\right\| .
\end{aligned}
$$

By Lemmas 2 and 3, we have

$$
\begin{aligned}
\left\|V_{N}\right\|_{L^{\infty}} & \leqslant C\left\|V_{N x}\right\|^{1 / 2}\left\|V_{N}\right\|^{1 / 2} \leqslant C\left(T_{0}\right), \\
\left\|V_{N t} E_{N t} E_{N t t}\right\|_{L^{1}} & \leqslant\left\|V_{N t}\right\|_{L^{\infty}}\left\|E_{N t}\right\|\left\|E_{N t t}\right\| \\
& \leqslant C\left\|V_{N t x}\right\|^{1 / 2}\left\|V_{N t}\right\|^{1 / 2}\left\|E_{N t}\right\|\left\|E_{N t t}\right\| \\
& \leqslant C\left\|V_{N t x}\right\|^{1 / 2}\left\|E_{N t t}\right\| .
\end{aligned}
$$

Substituting (43) and (44) into (42), we obtain

$$
\begin{aligned}
\frac{1}{2} \frac{d}{d t}\left\|E_{N t t}\right\|^{2} & \\
\leqslant & C\left\|E_{N t t}\right\|^{2}+C\left\|V_{N t x}\right\|^{1 / 2}\left\|E_{N t t}\right\|+C\left\|V_{N t t}\right\|\left\|E_{N t t}\right\| \\
& +C\left\|E_{N t t}\right\|^{2}+C\left\|E_{N t t}\right\|+C\left\|E_{N t t}\right\| \\
\leqslant & C\left\|E_{N t t}\right\|^{2}+C\left\|V_{N t x}\right\|+C\left\|V_{N t t}\right\|^{2}+C .
\end{aligned}
$$

Differentiating (14) in $t$, we have

$$
\left(V_{N t t t}, w_{j}\right)-\left(V_{N t x x}, w_{j}\right)=\left(\left(\left|E_{N}\right|^{2}\right)_{x x t}, w_{j}\right)+\left(G_{t}, w_{j}\right) .
$$

Multiplying (46) by $q_{j N}^{\prime \prime}(t)$ and summing up in $j$, we obtain

$$
\begin{aligned}
& \left(V_{N t t}, V_{N t t t}\right)-\left(V_{N t x x}, V_{N t t}\right) \\
& \quad=\left(\left(\left|E_{N}\right|^{2}\right)_{x x t}, V_{N t t}\right)+\left(G_{t}, V_{N t t}\right) .
\end{aligned}
$$

That is

$$
\begin{aligned}
\frac{1}{2} \frac{d}{d t} \| & V_{N t t}\left\|^{2}+\frac{1}{2} \frac{d}{d t}\right\| V_{N t x} \|^{2} \\
= & \left(\left(\left|E_{N}\right|^{2}\right)_{x x t}, V_{N t t}\right)+\left(G_{t}, V_{N t t}\right),
\end{aligned}
$$

where

$$
\begin{aligned}
&\left|G_{t}, V_{N t t}\right| \leqslant\left\|G_{t}\right\|\left\|V_{N t t}\right\| \leqslant C\left\|V_{N t t}\right\| \leqslant C\left\|V_{N t t}\right\|^{2}+C, \\
&\left(\left(\left|E_{N}\right|^{2}\right)_{x x t}, V_{N t t}\right) \\
&=\left(\left(E_{N} \overline{E_{N x x}}+\overline{E_{N}} E_{N x x}+2\left|E_{N x}\right|^{2}\right)_{t}, V_{N t t}\right) \\
& \leqslant\left\|E_{N t}\right\|_{L^{\infty}}\left(\left\|E_{N x x}\right\|^{2}+\left\|V_{N t t}\right\|^{2}\right) \\
&+\left\|E_{N}\right\|_{L^{\infty}}\left(\left\|E_{N t x x}\right\|^{2}+\left\|V_{N t t}\right\|^{2}\right) \\
&+2\left\|E_{N x}\right\|_{L^{\infty}}\left(\left\|E_{N t x}\right\|^{2}+\left\|V_{N t t}\right\|^{2}\right) \\
& \leqslant C\left\|E_{N t x}\right\|^{2}+C\left\|V_{N t t}\right\|^{4}+C\left\|E_{N t x x}\right\|^{2}+C .
\end{aligned}
$$

Differentiating (13) in $t$, we have

$$
\begin{aligned}
& i\left(E_{N t t}, w_{j}\right)+\left(E_{N t x x}, w_{j}\right) \\
& \quad=\left(\left(E_{N} V_{N}\right)_{t}, w_{j}\right)+\left(\left(E_{N} Q\right)_{t}, w_{j}\right) .
\end{aligned}
$$


Multiplying (51) by $\overline{p_{j N}^{\prime \prime}(t)}$ and summing up the above formulas for $j$ form 1 to $N$, we obtain

$$
\begin{aligned}
& i\left(E_{N t t}, E_{N t t}\right)+\left(E_{N t x x}, E_{N t t}\right) \\
& =\left(\left(E_{N} V_{N}\right)_{t}, E_{N t t}\right)+\left(\left(E_{N} Q\right)_{t}, E_{N t t}\right) .
\end{aligned}
$$

Taking the real parts of (52), we get

$$
\begin{aligned}
& \frac{1}{2} \frac{d}{d t}\left\|E_{N t x}\right\|^{2} \\
& \leqslant\left\|V_{N}\right\|_{L^{\infty}}\left(\frac{1}{2}\left\|E_{N t}\right\|^{2}+\frac{1}{2}\left\|E_{N t t}\right\|^{2}\right) \\
&+\left\|E_{N}\right\|_{L^{\infty}}\left(\frac{1}{2}\left\|V_{N t}\right\|^{2}+\frac{1}{2}\left\|E_{N t t}\right\|^{2}\right) \\
&+\|Q\|_{L^{\infty}}\left(\frac{1}{2}\left\|E_{N t}\right\|^{2}+\frac{1}{2}\left\|E_{N t t}\right\|^{2}\right) \\
&+\left\|Q_{t}\right\|_{L^{\infty}}\left(\frac{1}{2}\left\|E_{N}\right\|^{2}+\frac{1}{2}\left\|E_{N t t}\right\|^{2}\right) \\
& \leqslant C\left\|V_{N}\right\|_{L^{\infty}}\left\|E_{N t t}\right\|^{2}+C\left\|V_{N}\right\|_{L^{\infty}}+C\left\|E_{N t t}\right\|^{2}+C .
\end{aligned}
$$

Multiplying (26) by $-\lambda_{j} \overline{p_{j N}^{\prime}(t)}$ and summing up in $j$, we obtain

$$
\begin{aligned}
& i\left(E_{N t t}, E_{N t x x}\right)+\left(E_{N t x x}, E_{N t x x}\right) \\
& =\left(\left(E_{N} V_{N}\right)_{t}, E_{N t x x}\right)+\left(\left(E_{N} Q\right)_{t}, E_{N t x x}\right), \\
& \left\|E_{N t x x}\right\|^{2} \\
& =\left(\left(E_{N} V_{N}\right)_{t}, E_{N t x x}\right)+\left(\left(E_{N} Q\right)_{t}, E_{N t x x}\right) \\
& \quad-i\left(E_{N t t}, E_{N t x x}\right) \\
& \leqslant 5\left(\left\|E_{N t} V_{N}\right\|^{2}+\left\|E_{N} V_{N t}\right\|^{2}+\left\|E_{N t} Q\right\|^{2}\right. \\
& \left.\quad+\left\|E_{N} Q_{t}\right\|^{2}+\left\|E_{N t t}\right\|^{2}\right) .
\end{aligned}
$$

By the above lemmas, we have

$$
\begin{aligned}
\left\|E_{N t} V_{N}\right\|^{2} & \leqslant \frac{1}{2}\left\|E_{N t}\right\|_{L^{4}}^{4}+\frac{1}{2}\left\|V_{N}\right\|_{L^{4}}^{4} \\
& \leqslant C\left\|E_{N t x}\right\|\left\|E_{N t}\right\|^{3}+C\left\|V_{N x}\right\|\left\|V_{N}\right\|^{3} \\
& \leqslant C\left\|E_{N t x}\right\|+C\left\|V_{N x}\right\|^{4} \leqslant C\left\|E_{N t x}\right\|+C, \\
\left\|E_{N} V_{N t}\right\|^{2} & \leqslant \frac{1}{2}\left\|E_{N}\right\|_{L^{4}}^{4}+\frac{1}{2}\left\|V_{N t}\right\|_{L^{4}}^{4} \\
& \leqslant C\left\|E_{N x}\right\|\left\|E_{N}\right\|^{3}+C\left\|V_{N t x}\right\|\left\|V_{N t}\right\| \\
& \leqslant C\left\|v_{N t x}\right\|+C, \\
\left\|E_{N t} Q\right\|^{2} \leqslant & \frac{1}{2}\left\|E_{N t}\right\|_{L^{4}}^{4}+\frac{1}{2}\|Q\|_{L^{4}}^{4} \\
\leqslant & C\left\|E_{N t x}\right\|\left\|E_{N t}\right\|^{3}+C \leqslant C\left\|E_{N t x}\right\|+C, \\
& \left\|E_{N} Q\right\|_{t}\left\|^{2} \leqslant\right\| E_{N}\left\|_{L^{\infty}}^{2}\right\| Q_{t} \|^{2} \leqslant C .
\end{aligned}
$$

Substituting (55)-(58) into (54), we have

$$
\left\|E_{N t x x}\right\|^{2} \leqslant C\left\|E_{N t x}\right\|+C\left\|V_{N t x}\right\|+5\left\|E_{N t t}\right\|+C .
$$

Substituting (59) into (50), we get

$$
\begin{aligned}
\left(\left(\left|E_{N}\right|^{2}\right)_{x x t}, V_{N t t}\right) & \\
\leqslant & C\left\|E_{N t x}\right\|^{2}+C\left\|V_{N t t}\right\|^{4} \\
& +C\left(\left\|E_{N t x}\right\|+\left\|V_{N t x}\right\|+5\left\|E_{N t t}\right\|^{2}\right)+C \\
\leqslant & C\left\|E_{N t x}\right\|^{2}+C\left\|V_{N t t}\right\|^{4}+C\left\|V_{N t x}\right\|+\left\|E_{N t t x}\right\|^{2}+C .
\end{aligned}
$$

Substituting (49) and (60) into (48), we obtain

$$
\begin{aligned}
\frac{1}{2} \frac{d}{d t} \| & V_{N t t}\left\|^{2}+\frac{1}{2} \frac{d}{d t}\right\| V_{N t x} \|^{2} \\
& =\left(\left(\left|E_{N}\right|^{2}\right)_{x x t}, V_{N t t}\right)+\left(G_{t}, V_{N t t}\right) \\
& \leqslant C\left\|E_{N t x}\right\|^{2}+C\left\|V_{N t t}\right\|^{4}+C\left\|V_{N t x}\right\|+C\left\|E_{N t t x}\right\|^{2}+C .
\end{aligned}
$$

Combining (43), (45), (53), and (61), we have

$$
\begin{aligned}
\frac{1}{2} \frac{d}{d t}\left\|V_{N t t}\right\|^{2}+\frac{1}{2} \frac{d}{d t}\left\|V_{N t x}\right\|^{2}+\frac{1}{2} \frac{d}{d t}\left\|E_{N t t}\right\|^{2}+\frac{1}{2} \frac{d}{d t}\left\|E_{N t x}\right\|^{2} \\
\leqslant C\left(\left\|E_{N t x}\right\|^{2}+C\left\|V_{N t t}\right\|^{2}+C\left\|V_{N t x}\right\|^{2}+C\left\|E_{N t t}\right\|^{2}\right)^{2} \\
+C .
\end{aligned}
$$

That is

$$
\frac{d}{d t}\|\Psi\|^{2} \leqslant C\|\Psi\|^{4}+C
$$

Therefore, there exist a positive constant $T_{0}$, such that

$$
\|\Psi\|^{2} \leqslant C\left(T_{0}\right)
$$

for any $0 \leqslant t \leqslant T_{0}$, and $C$ is a positive constant that depends only on data and $T_{0}($ not on $N)$.

Multiplying (14) by $-\lambda_{j} q_{j N}(t)$ and then summing up the above formulas for $j$ form 1 to $N$, we obtain

$$
\begin{aligned}
\left(V_{N t t},\right. & \left.V_{N x x}\right)-\left(V_{N x x}, V_{N x x}\right) \\
& =\left(\left(\left|E_{N}\right|^{2}\right)_{x x}, V_{N x x}\right)+\left(G, V_{N x x}\right) .
\end{aligned}
$$

That is

$$
\left\|V_{N x x}\right\|^{2}=\left(V_{N t t}, V_{N x x}\right)-\left(\left(\left|E_{N}\right|^{2}\right)_{x x}, V_{N x x}\right)-\left(G, V_{N x x}\right),
$$


where

$$
\begin{aligned}
& \left(\left(\left|E_{N}\right|^{2}\right)_{x x}, V_{N x x}\right) \\
& \quad=2 \operatorname{Re} \int_{a}^{b} E_{N} \overline{E_{N x x}} V_{N x x} d x+2 \int_{a}^{b}\left|E_{N x}\right|^{2} V_{N x x} d x \\
& \quad \leqslant 2\left\|E_{N}\right\|_{L^{\infty}} \int_{a}^{b}\left|\overline{E_{N x x}} V_{N x x}\right| d x+2 \int_{a}^{b}\left|E_{N x}\right|^{2} V_{N x x} d x \\
& \quad \leqslant C\left\|E_{N x x}\right\|\left\|V_{N x x}\right\|+C\left\|\left|E_{N x}\right|^{2}\right\|\left\|V_{N x x}\right\| .
\end{aligned}
$$

This implies that

$$
\begin{gathered}
\left\|V_{N x x}\right\|^{2} \leqslant\left\|V_{N t t}\right\|\left\|V_{N x x}\right\|+C\left\|E_{N x x}\right\|\left\|V_{N x x}\right\| \\
+C\left\|\left|E_{N x}\right|^{2}\right\|\left\|V_{N x x}\right\|+\|G\|\left\|V_{N x x}\right\|, \\
\left\|V_{N x x}\right\| \leqslant\left\|V_{N t t}\right\|+C\left\|E_{N x}\right\|_{L^{4}}^{2}+C \leqslant\left\|V_{N t t}\right\|+C\left\|E_{N x}\right\|_{L^{4}}^{4}+C .
\end{gathered}
$$

By the above lemmas, we get

$$
\left\|E_{N x}\right\|_{L^{4}}^{4} \leqslant C\left\|E_{N x x}\right\|\left\|E_{N x}\right\|^{3} \leqslant C .
$$

Hence,

$$
\left\|V_{N x x}\right\| \leqslant C, \quad\left\|V_{N x x}\right\|^{2} \leqslant C .
$$

This completes the proof.

Lemma 5 (see [23, Lemma 4]). Let $X \subset E \subset Y$ be Banach spaces. If the embedding $X \hookrightarrow E$ is compact, then the following embeddings are also compact:

(1) $L^{q}(0, T ; X) \cap\left\{\varphi \quad:(\partial \varphi / \partial t) \in L^{1}(0, T ; Y)\right\} \quad \hookrightarrow$ $L^{q}(0, T ; E), 1 \leqslant q \leqslant \infty$,

(2) $L^{\infty}(0, T ; X) \cap\left\{\varphi \quad:(\partial \varphi / \partial t) \in L^{r}(0, T ; Y)\right\} \hookrightarrow$ $C([0, T] ; E), 1<r \leqslant \infty$.

Theorem 6. Under the conditions of Lemma 4, then there exists a local strong solution for (6)-(9).

Proof. According to those estimates outlined in the above lemmas, we know that $\left\|E_{N t}\right\|,\left\|V_{N t}\right\|,\left\|E_{N x x}\right\|$, and $\left\|V_{N x x}\right\|$ are all bounded uniformly in $N$. By the principle of compactness, there exist subsequences of $\left\{E_{N t}\right\},\left\{V_{N t}\right\},\left\{E_{N x x}\right\}$, and $\left\{V_{N x x}\right\}$ (we still denote these by the same letter) such that

$$
\begin{gathered}
E_{N t} \rightarrow E_{t}, \quad V_{N t} \rightarrow V_{t}, \\
E_{N x x} \rightarrow E_{x x}, \quad \Delta_{N x x} \rightarrow V_{x x} \quad \text { weakly. }
\end{gathered}
$$

Since $\left\|E_{N x}\right\|,\left\|V_{N x}\right\|$ are uniformly bounded in $N$, $H^{1}(\Omega) \hookrightarrow L^{2}(\Omega) \hookrightarrow H^{-1}(\Omega)$, we can choose subsequences of $\left\{E_{N}\right\},\left\{V_{N}\right\}$ (we still denote these by the same index) such that

$$
E_{N} \longrightarrow E, \quad V_{N} \longrightarrow V, \quad \text { strongly. }
$$

Therefore,

$$
E_{N} V_{N} \longrightarrow E V, \quad \text { strongly in } L^{\infty}\left(0, T_{0} ; L^{2}(\Omega)\right) .
$$

In fact, we have

$$
\begin{aligned}
\left\|E_{N} V_{N}-E V\right\| & =\left\|E_{N} V_{N}-E_{N} V+E_{N} V-E V\right\| \\
& \leqslant\left\|E_{N}\right\|_{L^{\infty}}\left\|V_{N}-V\right\|+\left\|E_{N}-E\right\|\left\|V_{N}\right\|_{L^{\infty}} .
\end{aligned}
$$

Since

$$
\begin{aligned}
\left\|E_{N}\right\|_{L^{\infty}} \leqslant C, & \|V\|_{L^{\infty}} \leqslant C, \\
\left\|V_{N}-V\right\| \longrightarrow 0, & \left\|E_{N}-E\right\| \longrightarrow 0,
\end{aligned}
$$

we get

$$
\left\|E_{N} V_{N}-E V\right\| \longrightarrow 0 .
$$

On the other hand, since

$$
\left(\left|E_{N}\right|^{2}\right)_{x x} \rightarrow\left(|E|^{2}\right)_{x x}, \quad \text { weakly in } L^{\infty}\left(0, T_{0} ; L^{2}(\Omega)\right),
$$

and for all $\varphi \in C_{0}^{\infty}(\Omega), E_{N} \in L^{\infty}\left(0, T ; H^{2} \cap H_{0}^{1}(\Omega)\right)$, we see that

$$
\begin{aligned}
& \left(\left(\left|E_{N}\right|^{2}\right)_{x x}-\left(|E|^{2}\right)_{x x}, \varphi\right) \\
& \quad=\left(\left|E_{N}\right|^{2}-|E|^{2}, \varphi_{x x}\right) \\
& \quad=\int_{a}^{b}\left(\left|E_{N}\right|-|E|\right)\left(\left|E_{N}\right|+|E|\right) \varphi_{x x} d x \\
& \quad \leqslant\left\|\left(\left|E_{N}\right|+|E|\right)\right\|_{L^{\infty}}\left\|\varphi_{x x}\right\|_{L^{\infty}}\left\|\left(\left|E_{N}\right|-|E|\right)\right\| \\
& \quad \leqslant C\left(\int_{a}^{b}\left|\left(E_{N}-E\right)\right|^{2} d x\right)^{1 / 2}=C\left\|\left(E_{N}-E\right)\right\| \rightarrow 0 .
\end{aligned}
$$

As $C_{0}^{\infty}(\Omega)$ is dense in $L^{2}(\Omega)$, this establishes the local strong solutions and completes the proof of Theorem 6 .

\section{Existence and Uniqueness of a Global Strong Solution}

In the following, in order to obtain the global strong solution we will give a priori estimates for the solution which we get in Theorem 6.

Lemma 7. Assume that $E_{0}(x) \in L^{2}$, then

$$
\|E(t, x)\|^{2}=\left\|E_{0}(x)\right\|^{2}, \quad \forall t \geqslant 0 .
$$

Proof. Multiply both sides of (1) by $i \bar{E}$, integrate the equation on $(a, b)$, and then take the real parts. It is easy to see that $(d / d t)\|E\|^{2}=0$. This implies that $\|E\|^{2}$ is a conserved quantity in respect to time. Therefore, Lemma 7 is proved. 
Lemma 8. Assume that $E_{0}(x) \in H^{2} \cap H_{0}^{1}(\Omega), v_{0}(x) \in H_{0}^{1}(\Omega)$, $v_{1}(x) \in L^{2}(\Omega)$, and $Q(t, x) \in C^{2}\left(0, T ; H^{2}(\Omega)\right)$; then for $\forall T>$ $0, t \in[0, T]$, one has

$$
\begin{gathered}
\left\|E_{x}\right\|^{2} \leqslant C(T), \quad\|V\|^{2} \leqslant C(T), \\
\left\|V_{t}\right\|^{2}+\left\|E_{t}\right\|^{2}+\left\|V_{x}\right\|^{2}+\left\|E_{x x}\right\|^{2} \leqslant C(T),
\end{gathered}
$$

where $C$ is a positive constant that depends only on data and $T$.

Proof. From (7), we have

$$
(V+Q)_{t t}-(V+Q)_{x x}=\left(|E|^{2}\right)_{x x} .
$$

Integrating $(81)$ over $[0, t]$, we obtain

$$
(V+Q)_{t}=\int_{0}^{t}\left(V+Q+|E|^{2}\right)_{x x} d t+n_{1}(x) .
$$

Taking the inner product of $(6)$ and $\overline{E_{t}}$, we get

$$
i\left(E_{t}, E_{t}\right)+\left(E_{x x}, E_{t}\right)=\left((V+Q) E, E_{t}\right) .
$$

Taking the real parts of (83), it follows that

$$
\begin{gathered}
-\frac{1}{2} \frac{d}{d t}\left\|E_{x}\right\|^{2}=\frac{1}{2} \int_{a}^{b}(V+Q)\left(\frac{d}{d t}|E|^{2}\right) d x, \\
\frac{d}{d t}\left\|E_{x}\right\|^{2}=-\frac{d}{d t} \int_{a}^{b}(V+Q)|E|^{2} d x+\int_{a}^{b}|E|^{2}(V+Q)_{t} d x .
\end{gathered}
$$

Substituting (82) into (84), we have

$$
\begin{aligned}
\frac{d}{d t}( & \left.\left\|E_{x}\right\|^{2}+\int_{a}^{b}(V+Q)|E|^{2} d x\right) \\
& =\int_{a}^{b}|E|^{2}(V+Q)_{t} d x \\
& =\int_{a}^{b}|E|^{2}\left(\int_{0}^{t}\left(V+Q+|E|^{2}\right)_{x x} d t+n_{1}(x)\right) d x .
\end{aligned}
$$

By (82), we get

$$
\begin{aligned}
& \frac{1}{2} \frac{d}{d t} \int_{a}^{b}(V+Q)^{2} d x \\
& \quad=\int_{a}^{b}(V+Q)\left(\int_{0}^{t}\left(V+Q+|E|^{2}\right)_{x x} d t+n_{1}(x)\right) d x .
\end{aligned}
$$

Integrating by parts yields that

$$
\begin{aligned}
\frac{1}{2} \frac{d}{d t} \int_{a}^{b} & \left(\int_{0}^{t}\left(V+Q+|E|^{2}\right)_{x} d t\right)^{2} d x \\
= & -\int_{a}^{b}\left(\int_{0}^{t}\left(V+Q+|E|^{2}\right)_{x x} d t\right)\left(V+Q+|E|^{2}\right) d x \\
& +Q(t, b)\left(\int_{0}^{t}\left(V+Q+|E|^{2}\right)_{x} d t\right)(t, b) \\
& -Q(t, a)\left(\int_{0}^{t}\left(V+Q+|E|^{2}\right)_{x} d t\right)(t, a) .
\end{aligned}
$$

Combining (85), (86), and (87), we have

$$
\begin{aligned}
\frac{d}{d t}\left(\left\|E_{x}\right\|^{2}+\int_{a}^{b}(V+Q)|E|^{2} d x\right) \\
+\frac{1}{2} \frac{d}{d t} \int_{a}^{b}(V+Q)^{2} d x \\
\quad+\frac{1}{2} \frac{d}{d t} \int_{a}^{b}\left(\int_{0}^{t}\left(V+Q+|E|^{2}\right)_{x} d t\right)^{2} d x \\
=\int_{a}^{b}\left(V+Q+|E|^{2}\right) n_{1} d x \\
\quad+Q(t, b)\left(\int_{0}^{t}\left(V+Q+|E|^{2}\right)_{x} d t\right)(t, b) \\
\quad-Q(t, a)\left(\int_{0}^{t}\left(V+Q+|E|^{2}\right)_{x} d t\right)(t, a) .
\end{aligned}
$$

Integrating (88) over $[0, t]$, we obtain

$$
\begin{aligned}
\left\|E_{x}\right\|^{2}+ & \int_{a}^{b}(V+Q)|E|^{2} d x+\frac{1}{2} \int_{a}^{b}(V+Q)^{2} d x \\
& +\frac{1}{2} \int_{a}^{b}\left(\int_{0}^{t}\left(V+Q+|E|^{2}\right)_{x} d t\right)^{2} d x \\
= & \left\|E_{0 x}(x)\right\|^{2}+\int_{a}^{b} n_{0}(x)\left|E_{0}(x)\right|^{2} d x+\frac{1}{2} \int_{a}^{b} n_{0}^{2}(x) d x \\
& +\int_{0}^{t} \int_{a}^{b}\left(V+Q+|E|^{2}\right) n_{1}(x) d x d t+F(t),
\end{aligned}
$$

where

$$
\begin{aligned}
& F(t)= \int_{0}^{t}\left[Q(t, b)\left(\int_{0}^{t}\left(V+Q+|E|^{2}\right)_{x} d t\right)(t, b)\right. \\
&\left.-Q(t, a)\left(\int_{0}^{t}\left(V+Q+|E|^{2}\right)_{x} d t\right)(t, a)\right] d t \\
&=\int_{0}^{t} \int_{a}^{b} \frac{d}{d x}\left[Q(t, x)\left(\int_{0}^{t}\left(V+Q+|E|^{2}\right)_{x} d t\right)\right] d x d t \\
&=\int_{0}^{t} \int_{a}^{b} Q(t, x)\left(\int_{0}^{t}\left(V+Q+|E|^{2}\right)_{x x} d t\right) d x d t \\
&+\int_{0}^{t} \int_{a}^{b} Q_{x}(t, x)\left(\int_{0}^{t}\left(V+Q+|E|^{2}\right)_{x} d t\right) d x d t .
\end{aligned}
$$

Substituting (82) into (90), we have

$$
\begin{aligned}
F(t)= & \int_{0}^{t} \int_{a}^{b} Q(t, x)\left((V+Q)_{t}-n_{1}(x)\right) d x d t \\
& +\int_{0}^{t} \int_{a}^{b} Q_{x}(t, x)\left(\int_{0}^{t}\left(V+Q+|E|^{2}\right)_{x} d t\right) d x d t \\
= & \int_{a}^{b}\left[Q(t, x)(V+Q)-Q(0, x) n_{0}(x)\right. \\
& \left.-\int_{0}^{t} Q_{t}(t, x)(V+Q) d t\right] d x
\end{aligned}
$$




$$
\begin{aligned}
& -\int_{0}^{t} \int_{a}^{b} Q(t, x) n_{1}(x) d x d t \\
& +\int_{0}^{t} \int_{a}^{b} Q_{x}(t, x)\left(\int_{0}^{t}\left(V+Q+|E|^{2}\right)_{x} d t\right) d x d t
\end{aligned}
$$

Therefore,

$$
\begin{aligned}
& |F(t)| \leqslant \int_{0}^{t} \int_{a}^{b}\left|Q_{t}(t, x)\right|\left(\left.|V+Q+| E\right|^{2}-|E|^{2} \mid\right) d x d t \\
& \int_{a}^{b}\left|Q\left(V+Q+|E|^{2}-|E|^{2}\right)\right| d x \\
& +\int_{0}^{t} \int_{a}^{b}\left|Q_{x}(t, x)\right|\left(\left|\int_{0}^{t}\left(V+Q+|E|^{2}\right)_{x} d t\right|\right) d x d t \\
& +C \leqslant\|Q\|\left\|V+Q+|E|^{2}\right\|+\|Q\|_{L^{\infty}}\|E\|^{2} \\
& +C \int_{0}^{t}\left[\left\|V+Q+|E|^{2}\right\|^{2}+\|E\|^{2}\right. \\
& +C \leqslant \frac{1}{4}\left\|\left(V+Q+|E|^{2}\right)\right\|^{2} \\
& +C \int_{0}^{t}\left[\left\|V+Q+|E|^{2}\right\|^{2}+\left\|\int_{0}^{t}\left(V+Q+|E|^{2}\right)_{x} d t\right\|^{2}\right] d t \\
& +C .
\end{aligned}
$$

Since

$$
(V+Q)|E|^{2}+\frac{1}{2}(V+Q)^{2}=\frac{1}{2}\left(V+Q+|E|^{2}\right)^{2}-\frac{1}{2}|E|^{4},
$$

we have the following estimate after combining (89) and (92):

$$
\begin{aligned}
\left\|E_{x}\right\|^{2}+ & \frac{1}{2}\left\|\left(V+Q+|E|^{2}\right)\right\|^{2}+\frac{1}{2}\left\|\int_{0}^{t}\left(V+Q+|E|^{2}\right)_{x} d t\right\|^{2} \\
\leqslant & \frac{1}{2}\|E\|_{L^{4}}^{4}+\left|\int_{0}^{t} \int_{a}^{b}\left(V+Q+|E|^{2}\right) n_{1}(x) d x d t\right|+|F(t)| \\
\leqslant & \frac{C \alpha}{2}\left\|E_{x}\right\|^{2}+\frac{C}{2 \alpha}+\frac{1}{4}\left\|\left(V+Q+|E|^{2}\right)\right\|^{2} \\
& +C \int_{0}^{t}\left[\left\|V+Q+|E|^{2}\right\|^{2}\right. \\
& \left.+\left\|\int_{0}^{t}\left(V+Q+|E|^{2}\right)_{x} d t\right\|^{2}\right] d t+C .
\end{aligned}
$$

We may choose $\alpha$ such that $C \alpha<1$ to obtain

$$
\begin{array}{r}
\left\|E_{x}\right\|^{2}+\left\|V+Q+|E|^{2}\right\|^{2}+\left\|\int_{0}^{t}\left(V+Q+|E|^{2}\right)_{x} d t\right\|^{2} \\
\leqslant C \int_{0}^{t}\left[\left\|\left(V+Q+|E|^{2}\right)\right\|^{2}\right. \\
\left.+\left\|\int_{0}^{t}\left(V+Q+|E|^{2}\right)_{x} d t\right\|^{2}\right] d t+C \\
\leqslant C \int_{0}^{t}\left[\left\|E_{x}\right\|^{2}+\left\|\left(V+Q+|E|^{2}\right)\right\|^{2}\right. \\
\left.+\left\|\int_{0}^{t}\left(V+Q+|E|^{2}\right)_{x} d t\right\|^{2}\right] d t+C .
\end{array}
$$

By Groonwall's lemma, we get

$$
\begin{aligned}
\left\|E_{x}\right\|^{2} & +\left\|\left(V+Q+|E|^{2}\right)\right\|^{2}+\left\|\int_{0}^{t}\left(V+Q+|E|^{2}\right)_{x} d t\right\|^{2} \\
& \leqslant C(T)
\end{aligned}
$$

for all $t \in[0, T]$, for any given $T>0$.

This implies that

$$
\begin{gathered}
\|E\|_{L^{\infty}} \leqslant C\left\|E_{x}\right\|^{1 / 2}\|E\|^{1 / 2} \leqslant C(T), \\
\|E\|_{L^{4}} \leqslant C\left\|E_{x}\right\|^{1 / 4}\|E\|^{3 / 4} \leqslant C(T), \\
\|V\| \leqslant\left\|V+Q+|E|^{2}\right\|+\|Q\|+\|E\|_{L^{4}}^{2} \leqslant C(T), \\
\|V\|^{2} \leqslant C(T) .
\end{gathered}
$$

Taking the inner product of (6) and $\overline{E_{x x}}$, we have

$i\left(E_{t}, E_{x x}\right)+\left(E_{x x}, E_{x x}\right)=\left(E V, E_{x x}\right)+\left(E Q, E_{x x}\right)$.

By (98), (99), and Lemma 2, we obtain

$$
\begin{aligned}
\left\|E_{x x}\right\| & \leqslant\left\|E V+E Q-i E_{t}\right\| \leqslant\|E V\|+\|E Q\|+\left\|E_{t}\right\| \\
& \leqslant\|E\|_{L^{\infty}}(\|V\|+\|Q\|)+\left\|E_{t}\right\| \leqslant\left\|E_{t}\right\|+C(T) .
\end{aligned}
$$

Taking the inner product of (7) and $V_{t}$, we get

$$
\begin{gathered}
\left(V_{t t}, V_{t}\right)-\left(V_{x x}, V_{t}\right)=\left(\left(|E|^{2}\right)_{x x}, V_{t}\right)+\left(G, V_{t}\right), \\
\frac{1}{2} \frac{d}{d t}\left\|V_{t}\right\|^{2}+\frac{1}{2} \frac{d}{d t}\left\|V_{x}\right\|^{2}=\int_{a}^{b}\left(|E|^{2}\right)_{x x} V_{t} d x+\int_{a}^{b} G V_{t} d x .
\end{gathered}
$$

Differentiating (6) with respect to $t$ then taking the inner product of the result and $\overline{E_{t}}$, we have

$$
\begin{aligned}
i\left(E_{t t},\right. & \left.E_{t}\right)+\left(E_{t x x}, E_{t}\right) \\
& =\left(E_{t} V, E_{t}\right)+\left(E V_{t}, E_{t}\right)+\left(E_{t} Q, E_{t}\right)+\left(E Q_{t}, E_{t}\right) .
\end{aligned}
$$


Taking the imaginary parts of (105), we obtain

$$
\frac{1}{2} \frac{d}{d t}\left\|E_{t}\right\|^{2}=\operatorname{Im}\left(E V_{t}, E_{t}\right)+\operatorname{Im}\left(E Q_{t}, E_{t}\right) .
$$

Combining (104) and (106), we get

$$
\begin{aligned}
& \frac{d}{d t}\left(\left\|V_{t}\right\|^{2}+\left\|V_{x}\right\|^{2}+\left\|E_{t}\right\|^{2}\right) \\
&=\int_{a}^{b}\left(|E|^{2}\right)_{x x} V_{t} d x+\int_{a}^{b} G V_{t} d x \\
&+\operatorname{Im}\left(E V_{t}, E_{t}\right)+\operatorname{Im}\left(E Q_{t}, E_{t}\right) \\
&=\int_{a}^{b} V_{t}\left(2 \operatorname{Re} E \overline{E_{x x}}+2\left|E_{x}\right|^{2}\right) d x \\
&+\int_{a}^{b} G V_{t} d x+\operatorname{Im}\left(E V_{t}, E_{t}\right)+\operatorname{Im}\left(E Q_{t}, E_{t}\right) \\
& \leqslant C\left\|V_{t}\right\|\left(\left\|E_{t}\right\|+C\right)+C\left\|E_{x x}\right\|^{1 / 2}\left\|V_{t}\right\| \\
&+C+\frac{1}{2}\left\|V_{t}\right\|^{2}+C\left\|V_{t}\right\|\left\|E_{t}\right\|+C\left\|E_{t}\right\| \\
& \leqslant C\left\|V_{t}\right\|^{2}+C\left\|E_{t}\right\|^{2}+C \\
& \leqslant C\left(\left\|V_{t}\right\|^{2}+\left\|E_{t}\right\|^{2}+\left\|V_{x}\right\|^{2}\right)+C .
\end{aligned}
$$

By Gronwall's lemma, we obtain

$$
\left\|V_{t}\right\|^{2}+\left\|E_{t}\right\|^{2}+\left\|V_{x}\right\|^{2} \leqslant C(T) .
$$

From (95), we get

$$
\begin{gathered}
\left\|E_{x x}\right\| \leqslant\left\|E_{t}\right\|+C(T) \leqslant C(T), \\
\left\|E_{x x}\right\|^{2} \leqslant C(T) .
\end{gathered}
$$

This completes the proof of Lemma 8 . So, we have the following theorem.

Theorem 9. The solution given by Theorem 6 exists for all time, that is, $T=\infty$.

The proof of uniqueness is done via standard integral estimates, so, we omit it here.

\section{Acknowledgments}

This work is supported by Qing Lan and “333" Project of Jiangsu Province and the NSF of the Jiangsu Higher Education Committee of China (11KJA110001). It is supported by the Brachman Hoffman Small Grant and Wellesley College Faculty Award.

\section{References}

[1] G. Boling and S. Longjun, "The global existence and uniqueness of classical solutions of periodic initial boundary value problems of Zakharov equations," Acta Mathematicae Applicatae Sinica, vol. 5, no. 2, pp. 310-324, 1982 (Chinese).
[2] G. Boling, "The initial boundary value problem for generalized Zakharov system," Applied Mathematics-A Journal of Chinese Universities, vol. 9, no. 1, pp. 1-12, 1994 (Chinese).

[3] J. Colliander, "Wellposedness for Zakharov systems with generalized nonlinearity," Journal of Differential Equations, vol. 148, no. 2, pp. 351-363, 1998.

[4] T. Ozawa, K. Tsutaya, and Y. Tsutsumi, "Well-posedness in energy space for the Cauchy problem of the Klein-GordonZakharov equations with different propagation speeds in three space dimensions," Mathematische Annalen, vol. 313, no. 1, pp. 127-140, 1999.

[5] L. Yongsheng, "On the initial boundary value problem for two dimensional systems of Zakharov equations and of complexSchrödinger-real-Boussinesq equations," Journal of Partial Differential Equations, vol. 5, no. 2, pp. 81-93, 1992.

[6] C. E. Kenig, G. Ponce, and L. Vega, "On the zakharov and zakharov-schulman systems," Journal of Functional Analysis, vol. 127, no. 1, pp. 204-234, 1995.

[7] G. C. Papanicolaou, C. Sulem, P. L. Sulem, and X. P. Wang, "Singular solutions of the Zakharov equations for Langmuir turbulence," Physics of Fluids B, vol. 3, no. 4, pp. 969-980, 1991.

[8] L. Glangetas and F. Merle, "Existence of self-similar blowup solutions for Zakhrov equation in dimension two. Part I," Communications in Mathematical Physics, vol. 160, no. 1, pp. 173-215, 1994.

[9] G. Boling, "Initial boundary value problem for one class of system of multidimensional nonlinear Schrödinger-Boussinesq type equation," Journal of Mathematical Research and Exposition, vol. 8, no. 1, pp. 61-71, 1988.

[10] G. Boling and S. Longjun, "The global solution of initial value problem for nonlinear Schrödinger-Boussinesq equation in 3Dimensions," Acta Mathematicae Applicatae Sinica, vol. 6, no. 1, pp. 11-21, 1990.

[11] Z. Zhou, "Spectral method for Zakharov equation with periodic boundary conditions," Acta Mathematicae Applicatae Sinica, vol. 5, no. 3, pp. 279-288, 1989.

[12] G. Boling, "Global smooth solutions for the system of Zakharov equations in nonhonogeneous medium," Northeastern Mathematical Journal, vol. 6, no. 4, pp. 379-390, 1990.

[13] G. Boling and Y. Guangwei, "Global smooth solution for the Klein-Gordon-Zakharov equations," Journal of Mathematical Physics, vol. 36, no. 8, pp. 4119-4124, 1995.

[14] G. Boling, "On the initial boundary value problem for some more extensive Zakharov equations," Journal of Mathematics, vol. 7, no. 3, pp. 269-275, 1987 (Chinese).

[15] L. Yongsheng and G. Boling, "Attractor of dissipative radially symmetric Zakharov equations outside a ball," Acta Mathematicae Applicatae Sinica, vol. 27, pp. 803-818, 2004.

[16] G. Boling, "On global solution for a class of systems of multi-dimensional generalized Zakharov type equation," Acta Mathematicae Applicatae Sinica, vol. 10, no. 4, pp. 419-433, 1994.

[17] V. Masselin, "A result on the blow-up rate for the Zakharov system in dimension 3," SIAM Journal on Mathematical Analysis, vol. 33, no. 2, pp. 440-447, 2001.

[18] P. A. Robinson, D. L. Newman, and M. V. Goldman, “Threedimensional strong langmuir turbulence and wave collapse," Physical Review Letters, vol. 61, no. 6, pp. 702-705, 1988.

[19] R. O. Dendy, Plasma Dynamics, Oxford University Press, Oxford, UK, 1990.

[20] P. M. Bellan, Fundamentals of Plasmas Physics, Cambridge University Press, Cambridge, UK, 2006. 
[21] V. E. Zakharov, "The collapse of langmuir waves," Journal of Experimental and Theoretical Physics, vol. 35, pp. 908-914, 1972.

[22] L. Nirenberg, "On elliptic partial differential equations," Annali della Scuola Normale Superiore di Pisa, vol. 13, pp. 115-162, 1959.

[23] J. Simon, "Nonhomogeneous viscous incompressible fluids: existence of velocity, density, and pressure," SIAM Journal on Mathematical Analysis, vol. 21, no. 5, pp. 1093-1117, 1990. 


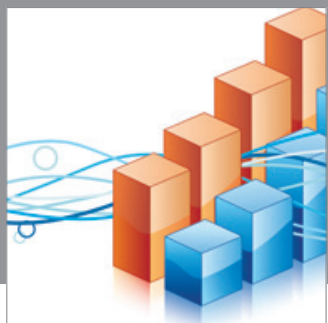

Advances in

Operations Research

mansans

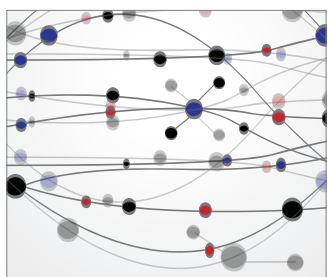

The Scientific World Journal
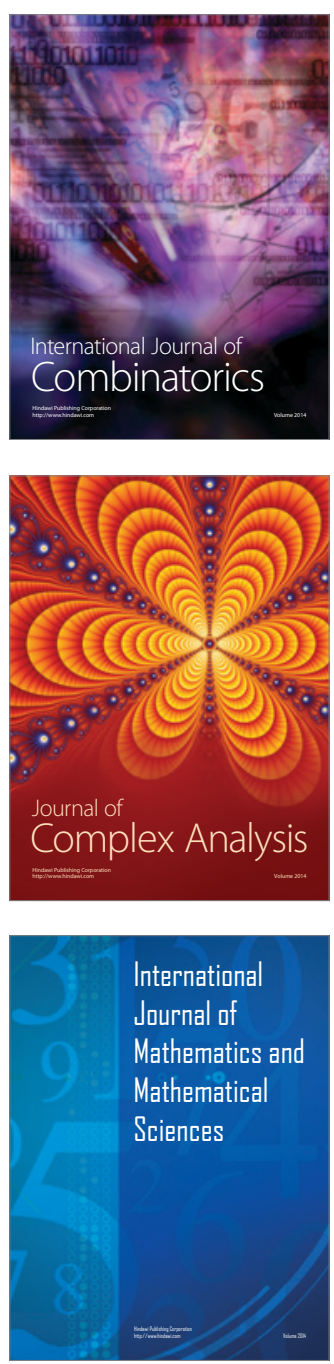
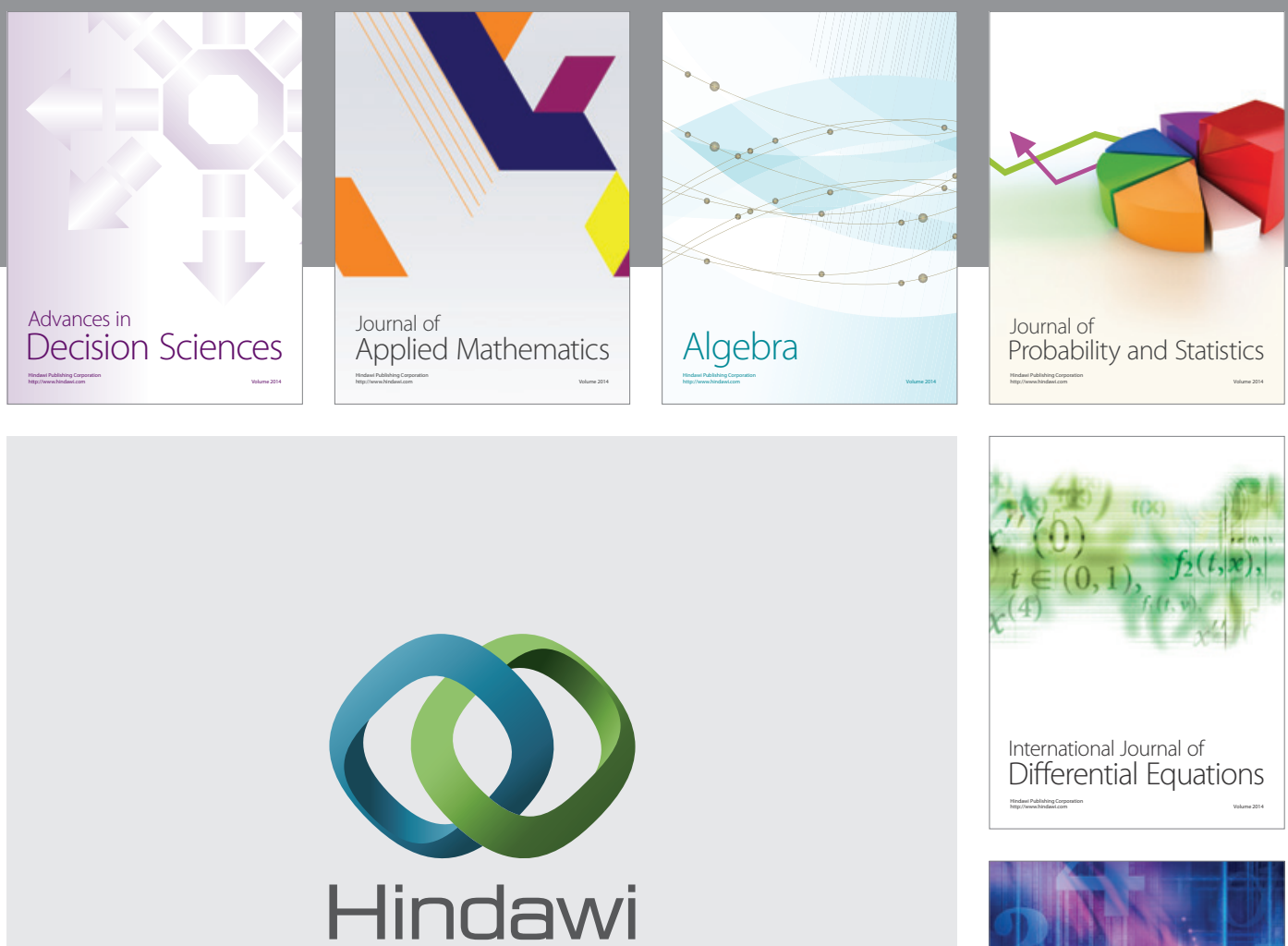

Submit your manuscripts at http://www.hindawi.com
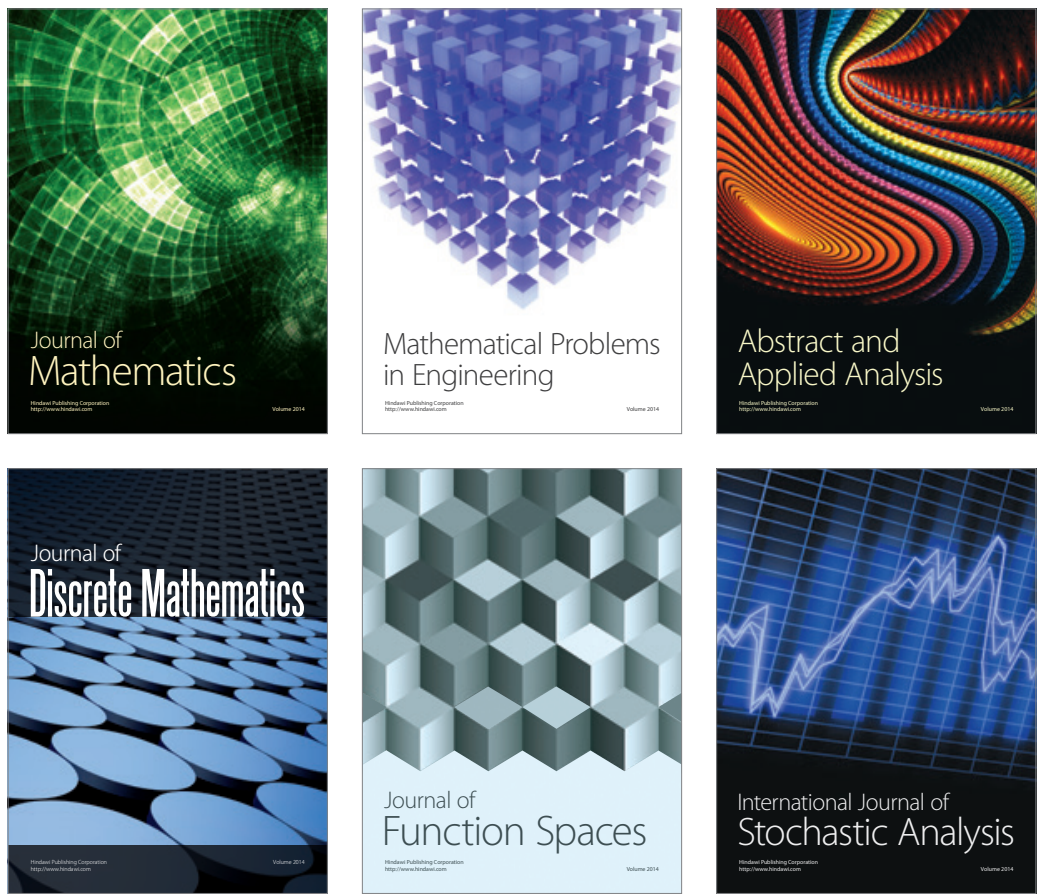

Journal of

Function Spaces

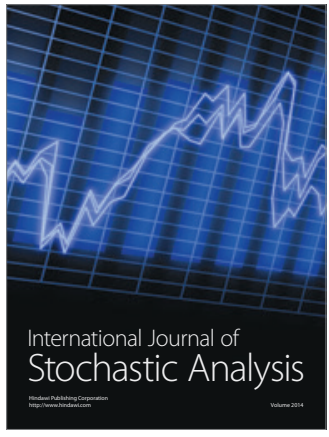

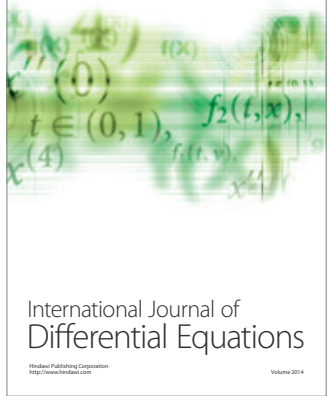
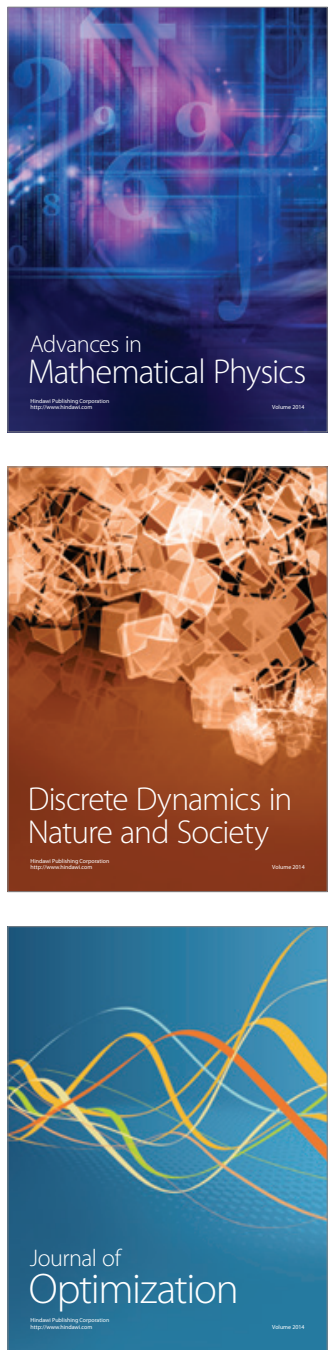